\title{
Seismic Wave Field Modeling with Graphics Processing Units
}

\author{
Tomasz Danek \\ Department of Geoinformatics and Applied Computer Science, \\ Faculty of Geology, Geophysics and Environmental Protection \\ AGH University of Science and Technology, \\ Al. Mickiewicza 30, 30-059 Krakow, Poland \\ tdanek@agh.edu.pl
}

\begin{abstract}
GPGPU - general-purpose computing on graphics processing units is a very effective and inexpensive way of dealing with time consuming computations. In some cases even a low end GPU can be a dozens of times faster than a modern CPUs. Utilization of GPGPU technology can make a typical desktop computer powerful enough to perform necessary computations in a fast, effective and inexpensive way. Seismic wave field modeling is one of the problems of this kind. Some times one modeled common shot-point gather or one wave field snapshot can reveal the nature of an analyzed wave phenomenon. On the other hand these kinds of modelings are often a part of complex and extremely time consuming methods with almost unlimited needs of computational resources. This is always a problem for academic centers, especially now when times of generous support from oil and gas companies have ended.
\end{abstract}

\section{Introduction}

Recent rapid development of computer based entertainment made graphics cards one of the most important part of whole computer systems. Typical modern personal computer systems and a constantly growing percentage of portable systems are designed to be multimedia centers. Fortunately some of a modern entertainment technology can be used for something more than an entertainment. It is well known that a graphic processing unit is in fact a powerful parallel system dedicated for matrix-to-matrix calculations (rendering). During last years many methods of using this power for calculations were developed. Intensive involvement of two the most important companies at this market: NVIDIA and ATI results in a marvelous dedicated software (e.g. CUDA, Stream SDK) and an astonishing hardware (NVIDIA TESLA). At the same time classic methods based on OpenGL including additions and standard hardware were developed. This paper is focused only on noncommercial, as free as possible, open-source solutions of the later kind. These solutions are usually better for long academic projects because rules of usage are clear and constant which is not always true in the case of commercial software, even if one can use them without any additional costs. One of the typical projects of this kind is a seismic wave field modeling. 
The growing popularity of this method in seismic and seismology (e.g. [12]) has been recently connected with the usage of inexpensive HPC clusters (e.g. [3]). But access to supercomputers or HPC clusters is usually limited. It is very common that small local problems, which can be easily solved with parallel computations have to be queued for hours because there are not enough free cluster resources. Moreover, many new extremely computationally expensive methods which use wave field modelings are being developed now. For example full wave form inversion through Monte Carlo sampling sometimes requires hundreds of thousands of models to be computed [2].

\section{Seismic Wave Field Modeling}

Wave field modeling is an important tool for seismic exploration and seismology. It can be used during all stages of seismic investigations and for various earthquake related analysis. First attempts of using a seismic wave field modeling were undertaken in the seventies by Alford, Kelly and others 45]. These attempts were limited to very small models due to the limitations of computers at that time. Even now serial computations for models of a standard exploration scale could last many days. Fortunately wave field modeling is a problem which is easy and effective to solve with parallel systems. In this paper a simple acoustic wave equation was chosen for a test computation:

$$
\frac{\partial^{2} p}{\partial t^{2}}-c^{2}\left(\frac{\partial^{2} p}{\partial x^{2}}+\frac{\partial^{2} p}{\partial z^{2}}\right)=f(x, z, t)
$$

where $p(x, z)$ is pressure, $c(x, z)$ is velocity of acoustic wave, $t$ is time and $f$ denotes function which describes pressure change in source.

The second order finite difference approximation of the above equation (without source term) can be written as follows [4]:

$$
\begin{aligned}
& p(i, j, k+1)= \\
& 2\left(1-2 \gamma^{2}\right) p(i, j, k)-p(i, j, k-1) \\
& +\gamma^{2}(p(i+1, j, k)+p(i-1, j, k)+p(i, j+1, k)+p(i, j-1, k))
\end{aligned}
$$

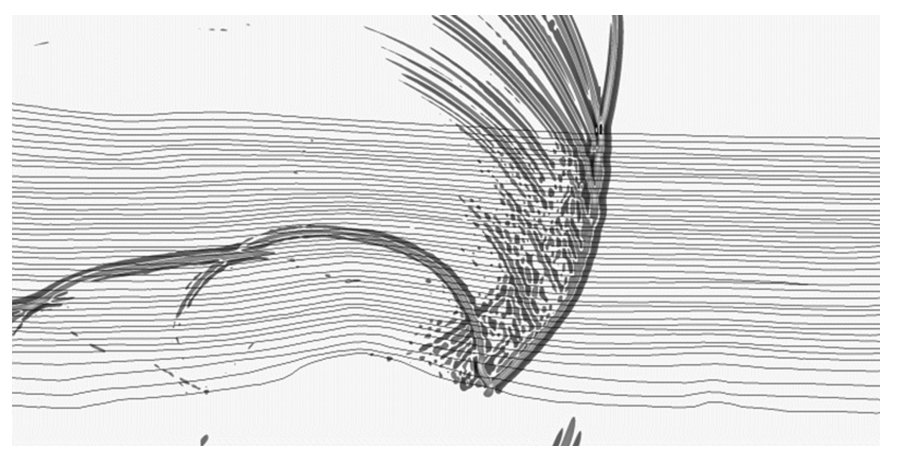

Fig. 1. Example result of acoustic wave field modeling 
where $\gamma=c \Delta t / \delta h, \Delta t$ is the time sampling interval, $\Delta h$ is the distance between the grid points in $\mathrm{x}$ and $\mathrm{z}$ directions. The stability criterion for the above scheme is: $\gamma \leq 1 / \sqrt{2}$.

It is also necessary to add proper border conditions. In this tests typical nonreflective boundary conditions by Reynolds [6 were used.

One of possible modeling result presentations, a snapshot of wave field propagating through complicated geological medium is presented in Figure 1.

\section{GPGPU}

GPGPU - General-Purpose computation on GPUs is one of the most rapidly developing part of modern computer science. Originally GPUs were created for very specific operations connected only with graphics. Later, when new advanced features - like possibility of floating point parallel operations - were introduced, it became clear that these units can be used for very fast and extremely inexpensive calculations. At the beginning this kind of utilization of GPUs seemed to be only marginal part of the IT market. But now, when the first GPU-based heterogeneous cluster joined the "Top 500", (November 17th, 2008) GPGPU is a mainstream technology with a sophisticated dedicated hardware and an effective commercial software. At the same time many free and open software solutions were created. They made a typical hardware to be much more programmerfriendly (e.g. BrookGPU).

The main source of computational power of a GPU is its design. Most of the transistors are dedicated to a data processing when the caching and data control functionalities are limited [7] (see Figure 2). This high specialization makes a GPU powerful tool for computations but at the same time makes it very weak for many other tasks which are hard to parallelize, like all kind of applications dominated by memory communication [8]. There are also other limitations like amount of memory and texture sizes. Usually all computational problems have to fit into $1 \mathrm{~GB}$ of memory and 4096 to 8192 texture size.
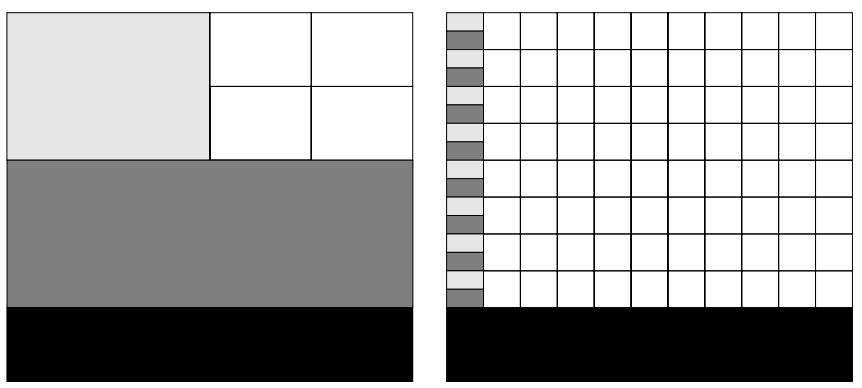

Fig. 2. Comparison of the design differences between CPU (left) and GPU (right) architecture [7]. (white: ALU; light gray: data control; dark gray: cache; black: DRAM). 


\section{Implementation}

The proposed solution was tested on one of the departmental PC cluster, made of 30 computers with low end AGP Nvidia adapters. The best ones were $7300 \mathrm{GT}$ with $256 \mathrm{MB}$ memory. Additional tests were done on PCI Express Nvidia 8600 GT card with $512 \mathrm{MB}$ memory. Other important card parameters are shown in table 1. All cluster machines were working under Linux operating system but all tests were repeated for MS Windows XP for comparison purposes. In both cases the newest possible standard drivers were used. All test codes have been written in $\mathrm{C}$ (gcc for Linux and $\mathrm{VC}++$ for Windows) and OpenGL shading language (Listing 1). Data were stored in GL_TEXTURE_RECTANGLE_ARB texture targets which are the most natural targets for computational purposes. It is because of a lack of coordinates normalization and an arbitrary dimensions. In all cases only one floating point number per texel was stored, therefore GL_LUMINANCE texture format and GL_FLOAT_R32_NV internal format were used. For intra-cluster communication mpich implementation of MPI was used. All test runs were done for a simple two layer geological model of various sizes. Wave velocity in upper layer was $1000 \mathrm{~m} / \mathrm{s}$ and $2000 \mathrm{~m} / \mathrm{s}$ in lower. Wave motion was modeled for 0.3 second. Distance between grid points was 1 meter

Table 1. Parameters of GPUs used in tests

\begin{tabular}{|c|c|c|c|c|c|c|}
\hline Model & Chip & $\begin{array}{c}\text { Core } \\
\text { clock }\end{array}$ & $\begin{array}{c}\text { Memory } \\
\text { clock }\end{array}$ & $\begin{array}{c}\text { Memory } \\
\text { type }\end{array}$ & $\begin{array}{c}\text { Pixel } \\
\text { shader }\end{array}$ & $\begin{array}{c}\text { Vertex } \\
\text { shader }\end{array}$ \\
\hline $7300 \mathrm{GT}$ & G73 & $400 \mathrm{MHz}$ & $1000 \mathrm{MHz}$ & $\begin{array}{c}\text { GDDR3 } \\
\text { (128Bit) }\end{array}$ & 8 & 4 \\
\hline $8600 \mathrm{GTS}$ & G84 & $675 \mathrm{MHz}$ & $2000 \mathrm{MHz}$ & $\begin{array}{c}\text { GDDR3 } \\
\text { (128Bit) }\end{array}$ & up to 32 & up to 32 \\
\hline
\end{tabular}

Listing 1. OpenGL shading language implementation of equation [2, vec2 type was used for code clarity. $V p$ - wave velocity; $p$ and $p m$ - wave field at time $t$ and $t-1 ; d s$ - distance between grid points; $d t r$ - time step.

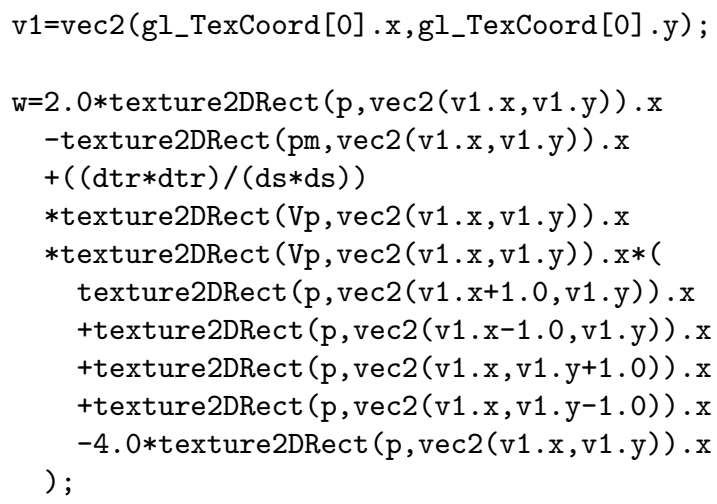


which means that 1200 iterations were needed for final result. In all numerical experiments non-reflective boundary conditions were used. In case of GPU computations these kind of conditions are applied by proper if statements which makes computations up to $10 \%$ slower. Alternatively attenuating boundary conditions can be used but they require an additional texture to be created, which is memory consuming. Additionally all models were calculated conventionally using typical cluster node with Intel Pentium $43.0 \mathrm{Ghz}$ processor and $2 \mathrm{~GB}$ of memory. In this part of the experiment GNU and Intel compilers were used. Obtained times were a base for unoptimized and optimized speedup calculations.

\section{Selected Results and Discussion}

In the experiment computational times needed by both of the analyzed GPUs were calculated for Linux and Windows operating systems. The relation between time of computations and model size is shown in Figure 3 . The results show that in 8600 GTS card case computations for the very small models are marginally faster under Linux system but for larger models Windows driver is faster. The interesting phenomenon is a rapid increase of the time of computations for the largest analyzed model under Linux. Exactly the same was observed for one of the older Windows drivers, so probably this problem will be fixed in the future. Surprisingly in the case of 7300 GT relation between results is reversed and computations under Linux are usually faster. Figures [4 5] and 6] show how much faster GPU computations are in comparison with unoptimized and optimized codes run on Intel P4 3.0 GHz CPU . It is clearly visible that for usual scale models GPU computations are 10 (7300 GT) to 50 (8600 GTS) times faster than gcc compiled CPU code with default set of flags (no optimization)(Figure 4). Results of similar experiment but for optimized gcc code are presented in Figure 5. The fastest code was obtained for singe -O3 flag. Executables compiled with more sophisticated options were slower or their average processor time was almost exactly the same. In this case GPU version was up to 20 times faster. The last comparison was done for Intel C compiler (Figure 6). As it was expected icc codes with standard set of flags and autoparallelization were better than those generated with gcc, but still 8600 GTS card was over a dozen times faster. It is important to emphasize that both cards are inexpensive and one can get the cheaper one for much less then 100 USD. The other important phenomenon to discuss is a rapid fall of the acceleration curve for larger model in the case of the 8600 GTS card. GPGPU calculations speedup curves usually have rather unstable shape which is strongly connected with the version of the card driver. It is also important to realize that analyzed case is special and very well suited for GPGPU and one should not expect similar speedups for other kinds of computations.

\section{Summary}

Possibility of GPGPU computations application in seismic wave field modeling was presented in this paper. It is clearly visible that graphics processing units 


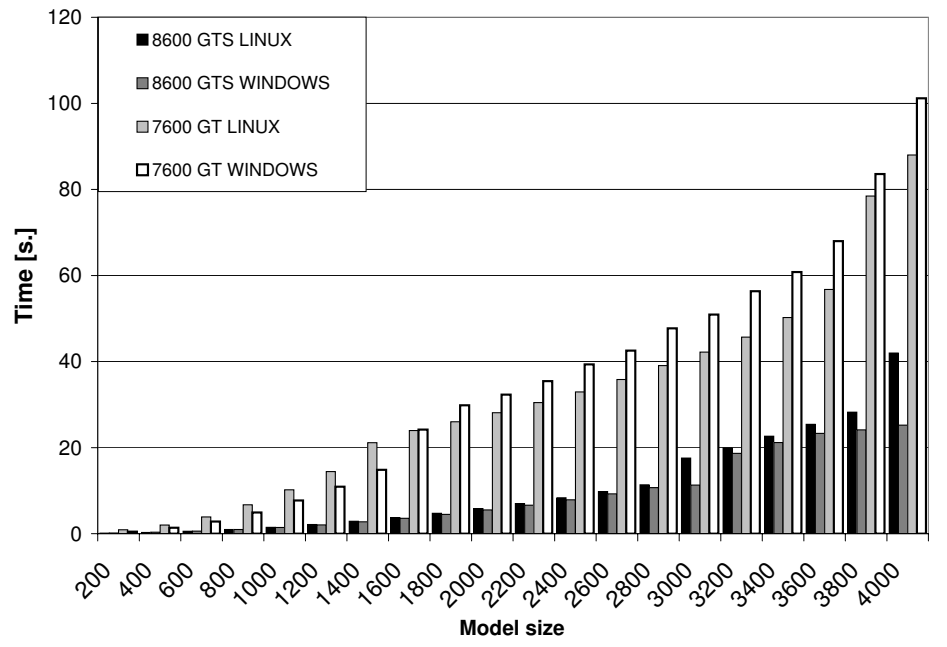

Fig. 3. Relation between time of computations and size of the model for selected hardware and systems. Values on bottom axis represent grid points per side of rectangular model. Black bars: 8600 GTS - Linux; dark gray bars: 8600 GTS - Windows; light gray bars: 7300 GT - Linux; white bars: 7300 GT - Windows.

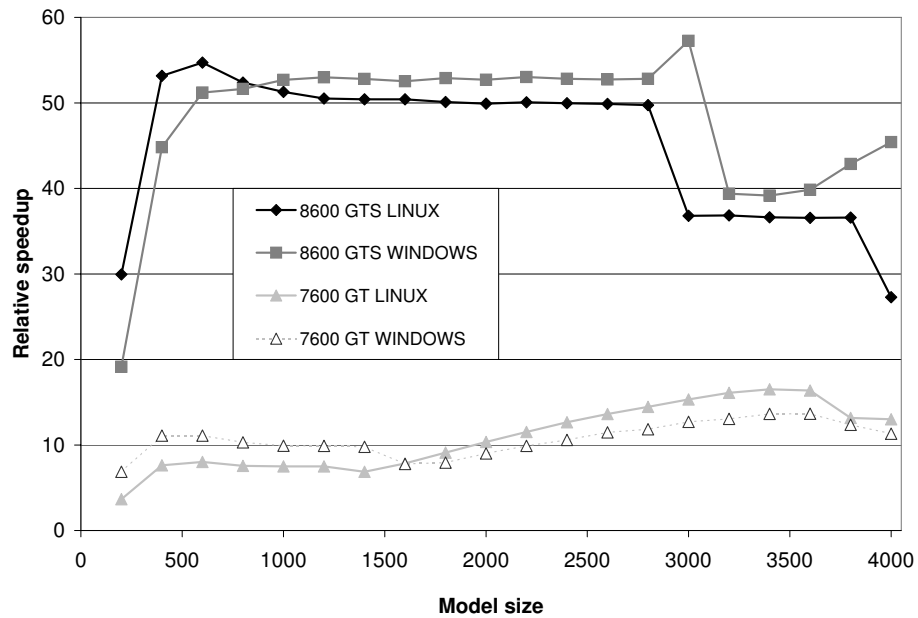

Fig. 4. Relative speedups for selected hardware and systems. The base for speedup calculations is time of conventional CPU computations performed on Intel Pentium 4 $3.0 \mathrm{GHz}$ processor - results for unoptimized gcc. Black rectangles: 8600 GTS - Linux; dark gray rectangles: 8600 GTS - Windows; light gray triangles: 7300 GT - Linux; white triangles: $7300 \mathrm{GT}$ - Windows. 


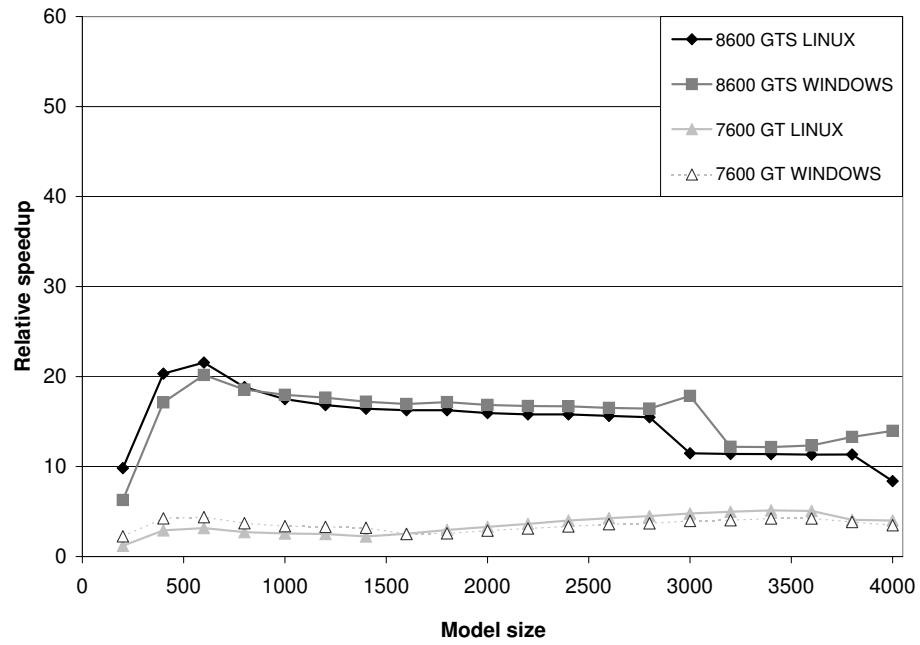

Fig. 5. Relative speedups for selected hardware and systems. The base for speedup calculations is time of conventional CPU computations performed on Intel Pentium 4 $3.0 \mathrm{GHz}$ processor - results for the best set of gcc optimization options. Black rectangles: 8600 GTS - Linux; dark gray rectangles: 8600 GTS - Windows; light gray triangles: 7300 GT - Linux; white triangles: 7300 GT - Windows.

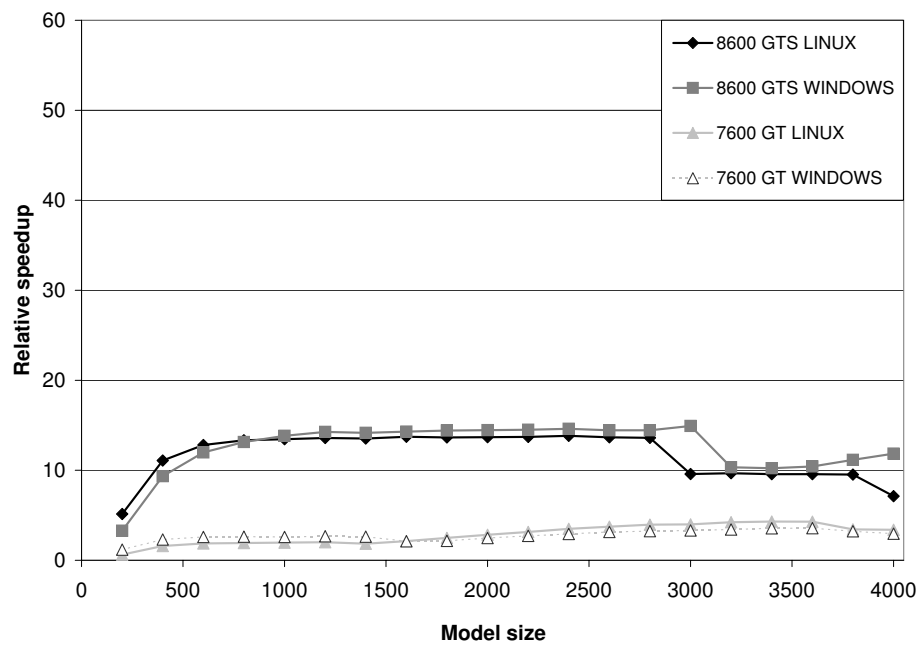

Fig. 6. Relative speedups for selected hardware and systems. The base for speedup calculations is time of conventional CPU computations performed on Intel Pentium 4 $3.0 \mathrm{GHz}$ processor - results for the best set of Intel compiler (icc) optimization options. Black rectangles: 8600 GTS - Linux; dark gray rectangles: 8600 GTS - Windows; light gray triangles: 7300 GT - Linux; white triangles: 7300 GT - Windows. 
are very effective in this kind of computations. It should be stressed that presented tests are preliminary and other more complicated cases must be studied. Anyway the obtained results are promising and can be a contribution to studies concerning efficiency of alternative modern HPC methods in seismic and seismology.

Acknowledgments. This work was financed by the AGH - University of Science and Technology, Faculty of Geology, Geophysics and Environmental Protection as a part of statutory project number 11.11.140.561.

\section{References}

1. Pietsch, K., Marzec, P., Kobylarski, M., Danek, T., Lesniak, A., Tatarata, A., Gruszczyk, E.: Identification of seismic anomalies caused by gas saturation on the basis of theoretical P and PS wavefield in the Carpathian Foredeep, SE Poland. Acta Geophysica 55(2) (2007)

2. Debski, W., Danek, T., Pieta, A., Lesniak, A.: Waveform inversion through the Monte Carlo sampling. In: 31st General assembly of the European Seismological Commission, Hersonissos, Crete (2008)

3. Lesniak, A., Danek, T.: Efficiency of Linux clusters in multi-component elastic wave field modeling in anisotropic media. In: 68th EAGE conference and exhibition, Wien (2006)

4. Alford, R.M., Kelly, K.R., Boore, D.M.: Accuracy of finite - difference modeling of acoustic wave propagation. Geophysics 39(6) (1974)

5. Kelly, K.R., Ward, R.W., Treitel, S., Kelly, K.R., Alford, R.M.: Synthetic seismograms: A finite-difference approach. Geophysics 41(6) (1975)

6. Reynolds, A.C.: Boundary conditions for the numerical solution of wave propagation problems. Geophysics 43 (1978)

7. NVIDIA CUDA Compute Unified Device Architecture Programming Guide. Version 2.0 - 06. 07 (2008)

8. Owens, J.D., Luebke, D., Govindaraju, N., Harris, M., Kruger, J., Lefohn, A.E., Purcell, T.J.: A Survey of General-Purpose Computation on Graphics Hardware. Computer Graphics forum 26(1) (2007) 